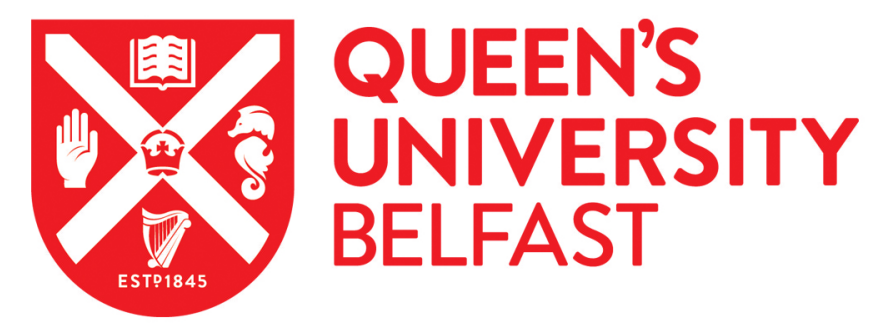

\title{
Effects of TAT-SOD at Acupoints on Essential Hypertension by Monitoring Meridians Electrical Potential
}

Guo, J., Xu, J., Chen, T., Xu, M., Liu, S., Zhang, C., Ke, L., Zhou, J., Wang, Q., \& Rao, P-F. (2019). Effects of TAT-SOD at Acupoints on Essential Hypertension by Monitoring Meridians Electrical Potential. Chinese journal of integrative medicine. https://doi.org/10.1007/s11655-019-3173-9

\section{Published in:}

Chinese journal of integrative medicine

\section{Document Version:}

Peer reviewed version

Queen's University Belfast - Research Portal:

Link to publication record in Queen's University Belfast Research Portal

\section{Publisher rights}

(c) The Chinese Journal of Integrated Traditional and Western Medicine Press and Springer-Verlag GmbH Germany, part of Springer Nature 2019. This work is made available online in accordance with the publisher's policies. Please refer to any applicable terms of use of the publisher.

\section{General rights}

Copyright for the publications made accessible via the Queen's University Belfast Research Portal is retained by the author(s) and / or other copyright owners and it is a condition of accessing these publications that users recognise and abide by the legal requirements associated with these rights.

Take down policy

The Research Portal is Queen's institutional repository that provides access to Queen's research output. Every effort has been made to ensure that content in the Research Portal does not infringe any person's rights, or applicable UK laws. If you discover content in the Research Portal that you believe breaches copyright or violates any law, please contact openaccess@qub.ac.uk. 


\section{Title Page}

\section{Monitoring Effects of TAT-SOD at Acupoints on Essential \\ Hypertension by Measuring Meridians Electrical Potential}

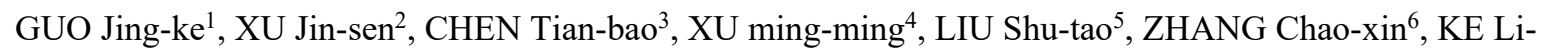
jing ${ }^{1}$, ZHOU Jian-wu ${ }^{1}$, WANG Qiang ${ }^{7}$, RAO Ping-fan ${ }^{1}$

1. Shanghai Institutes for Biological Sciences Chinese Academy of Sciences-Zhejiang Gongshang University Joint Centre for Food and Nutrition Research, Zhejiang Gongshang University, Hangzhou (310035), China; 2. Acupuncture College, Fujian University of Traditional Chinese Medicine, Fuzhou (350003), China; 3. Natural Drug Discovery Group, School of Pharmacy, Queen's University, Belfast (BT97BL), UK; 4. College of Oceanology and Food Science, Quanzhou Normal University, Quanzhou (362000), China; 5. College of Biological Science and Technology, Fuzhou University, Fuzhou (350108), China; 6. Guangzhou Halcyon Technology Ltd., Guangzhou (510630), China; 7. Institute of Food Science and Technology, Chinese Academy of Agricultural Sciences, Beijing (100000) China.

Correspondence to: Prof. Rao Ping-Fan, No. 149, Jiaogong Road, Hangzhou, China, Tel: 8613750842288 ; pingfan.rao@gmail.com.

Supported by National Key R\&D Program of China (No. 2016YFD0400202), National Natural Science Foundation of China (No. 31500685, 30973720), Zhejiang Provincial Natural Science Foundation of China (No. LY16C050002), Start-Up Fund of Research in Quanzhou Normal University (No. G16062). 


\begin{abstract}
Objectives:

Our previous study demonstrates that different antioxidant interventions at Zusanli (ST36) resulted in different patterns of electrical potential difference (EPD) between acupoints, providing the evidence that EPD between acupoints may reflect the conduction of superoxide anion's along the meridian. This study aimed to validate the discovery by investigating the effect on essential hypertension of the topical application of TAT-SOD at left acupoint Zusanli (ST36) and observe whether the change of EPD can be related to the change of blood pressure.
\end{abstract}

Methods: The study design called for at least 16 evaluable essential hypertension and 16 healthy subjects. Firstly, EPD between the left acupoints of Yanglingquan (GB34) and Qiuxu (GB40) was screened out for the EPD detection. An intracellular superoxide quenching enzyme, TAT-SOD was topically applied to the acupoint of Zusanli (ST36) within an area of $1 \mathrm{~cm}^{2}$ once a day, and the influence on EPD was investigated. The dosage applied to TAT-SOD group $(\mathrm{n}=8)$ was $0.2 \mathrm{ml}$ of $3000 \mathrm{U} / \mathrm{ml}$ TAT-SOD cream prepared by adding purified TAT-SOD to a vehicle cream, while placebo group $(n=8)$ used the vehicle cream instead. The left acupoints of Yanglingquan (GB34) and Qiuxu (GB40) were selected for EPD measurement after comparing EPD readings between 5 acupoints on each of all 12 meridians.

Results: EPDs between the left acupoints of Yanglingquan (GB34) and Qiuxu (GB40) for 16 patients of evaluable essential hypertension and 16 healthy subjects were $44.9 \pm 6.4 \mathrm{mV}$ and $5.6 \pm 0.9 \mathrm{mV}$, respectively. Daily application of TAT-SOD for 15 days at Zusanli (ST36) of essential hypertension patients significantly decreased SBP and DBP of $179.6 \mathrm{mmHg}$ and $81.5 \mathrm{mmHg}$ to $153.1 \mathrm{mmHg}$ and $74.1 \mathrm{mmHg}$, respectively. Responding to the change in blood pressure, EPD between the left acupoints of Yanglingquan (GB34) and Qiuxu (GB40) also declined from $44.4 \mathrm{mV}$ to $22.8 \mathrm{mV}$ with the same trend. No change was observed with SBP, DBP and EPD between the left acupoints of Yanglingquan (GB34) - Qiuxu (GB40) with the daily application of the placebo cream.

\title{
Conclusions:
}

Enzymatic scavenging of the intracellular superoxide at Zusanli (ST36) proved to be effective in decreasing SBP and DBP. Results reconfirm the involvement of superoxide anions and its transportation along the meridians, and demonstrate that EPD between acupoints may be an indicator to reflect its functioning status. Moreover, preliminary results suggest a close correlation between EPD readings and blood pressure readings, implying a possibility of using EPD as a sensitive parameter to determine blood pressure and to monitor the treatment.

\section{Key words}

TAT-SOD, reactive oxygen species, electrical potential, essential hypertension, acupoints 
Acupuncture meridians are considered to be channels for bio-energy (Qi), directing blood flow and connecting organs to body surface as well as all parts of the body in Traditional Chinese Medicine (TCM) theory. They connect visceral organs with acupoints located along the meridian lines. Acupuncture meridians and points are also a basic element in the diagnosis and treatment of illness ${ }^{[1]}$. For decades, electrical devices were used to detect and monitor this bio-energy in acupuncture meridians and points. The first efforts for the electrical detection of meridians and acupoints can be dated back to the 1950s, when Reinhard Voll ${ }^{[2]}$ and Yoshio Nakatani ${ }^{[3]}$ discovered some acupoints were with unique electrical characteristics and they were related to the health condition of a person. Since then, numerous studies have elaborated the electrical properties of acupoints and meridians. These properties include increased conductance, reduced impedance and resistance, increased capacitance, and elevated electrical potential compared to non-acupoints and non-meridians, demonstrating the electric nature of meridians ${ }^{[4,5]}$.

Superoxide is one of the main reactive oxygen species (ROS), a product of normal cellular metabolism, which acts as a messenger not only in intracellular, but also intercellular signaling processes involved in activation of proinflammatory responses and mediate degradation of aggregan and collagen ${ }^{[6]}$. Tamas Dolowschiak indicated an important role of superoxide in horizontal epithelial cell-cell communication ${ }^{[7]}$. Wibke Bechtel observed modulation of intercellular superoxide signaling of human tumor cells ${ }^{[8]}$. Since superoxide is negatively charged, its signaling effect is of electric nature. In our studies of superoxide distribution in living SD-rats by ROS indicator visualization, a few vertical green fluorescent lines were demonstrated on the frontal abdominal wall. They were related to neither veins nor nerves but could be almost perfectly superimposable on a standard human acupuncture meridian network ${ }^{[9]}$. Moreover, topical application of TAT-Cu, $\mathrm{Zn}$-superoxide dismutase (TAT-SOD) to acupoints were found to result in acupuncture-like action for treating simple obesity ${ }^{[10,11]}$ and allergic rhinitis ${ }^{[12]}$. TAT-SOD is a recombinant protein of human liver $\mathrm{Cu}, \mathrm{Zn}$-superoxide dismutase (SOD) fused with TAT peptide, which enables the fusion protein to be delivered cross the cell membrane while maintaining SOD activities, making it an intracellular superoxide quenching enzyme ${ }^{[13]}$. Furthermore, TAT-SOD was found to be delivered not only into cells ${ }^{[14,15]}$ but also into mitochondria where superoxide is generated ${ }^{[16]}$, suggesting a great potential of TAT-SOD as an ideal intracellular anti-oxidant solution. It was found that TAT-SOD could increase the viability of mammalian cells irradiated by UV-C ${ }^{[14]}$ and $U V-B^{[17]}$, prevented and protected ${ }^{[18]}$ the skins of guinea pigs damaged by single dose and repeated UV-B radiation. All those results point to a new applaudable hypothesis of meridians as channels of superoxide and superoxide modulation along meridians as acupuncture mechanism.

Due to its negative charge, superoxide migration is driven by the voltage difference. Therefore, any antioxidant intervention at acupoints is possible to be monitored by the electrical potential difference (EPD) between acupoints along the meridians. The investigation of the effects of acupoints antioxidant interventions on the 
meridian EPD confirms superoxide's transportation along the meridians and demonstrates the feasibility of acupoints EPD measurement as a tool to monitor changes in the meridians and acupoints ${ }^{[19,20]}$.

Essential hypertension is considered to be one of the most relevant cardiovascular risk factors and is an important cause of mortality and morbidity around the world. Acupuncture in Stomach Meridian is effective in lowering blood pressure and curing original symptoms ${ }^{[21,22]}$. A large amount of evidences show that superoxide anions play a central role in its pathophysiology ${ }^{[23]}$, leading to the development of new therapeutic strategies against hypertension based on the use of antioxidants agents ${ }^{[24,25]}$.

This study aims to validate our previous discovery by investigating the relationship between blood pressure and the EPD of acupoints and observing whether the change of EPD can be related to the change of blood pressure as the result of the topical application of TAT-SOD at acupoint Zusanli (ST36).

\section{MATERIALS AND METHODS}

\section{TAT-SOD expression}

TAT-SOD was prepared by recombinant expression of a fusion protein of human $\mathrm{Cu}, \mathrm{Zn}$-SOD fused with TAT peptide in E. coli as follows: Constructs preparation: The nucleic acid sequence encoding TAT-SOD fusion protein was constructed by DNA recombinant technology and inserted into expression vector pGEX-2T; Cell culture and transfections: E. coli (BL21, DH5a) cells were transformed with the expression vector pGEX-2T containing the inserted TAT-SOD; TAT-SOD fusion protein preparation: TAT-SOD was expressed in the E. coli by the induction of isopropyl- $\beta$-D-thiogalactoside (IPTG) and prepared by affinity chromatography to electrophoretically pure for use ${ }^{[13]}$. TAT-SOD cream was prepared by the homogenization of the isolated TATSOD with the vehicle cream, which contains water, propylene glycol, myristyl myristate, glyceryl stearate, oleic acid, stearic acid. TAT-SOD in the cream is remarkably stable. SOD activity loss was less than $5.6 \%$ when TAT-SOD was stored at room temperature for 6 months ${ }^{[26]}$.

\section{Human Subjects}

The study design called for at least 16 evaluable essential hypertension and 16 healthy subjects. All participants were volunteers and recruited from those referred to Hospital of Fujian Traditional Chinese Medicine University between September 1, 2012, and July 1, 2013. Written informed consent was obtained from all subjects before enrollment and all subjects were free to withdraw from the study at any time. The Medical Research Ethics Committee and Institutional Review Board of Fujian Institute of Traditional Chinese Medicine provided approval for this study (30973720), and all subjects gave informed consent for publication of these data. After washout of antihypertensive medication for 4 weeks, all subjects showed mild to moderate hypertension (> 140/90 mmHg and diastolic blood pressures lower than $110 \mathrm{mmHg}$ ). Exclusion criteria were as follows: 1) Age under 40 or over 70,2) being pregnant or planning pregnancy, 3) secondary hypertension, 4) 
malignant or accelerated hypertension, 5) nephrotic syndrome, 6) history of stroke within 3 months, 7) history of acute myocardial infarction or unstable angina within 3 months, 8) congestive heart failure, 9) renal dysfunction, 10) hepatic dysfunction, 11) dementia, 12) alcoholics or other drug abusers, 13) inability to maintain medication during the study period, 14) failure to obtain informed consent ${ }^{[27]}$.

\section{Selection of Electric Potential Difference Detection Points}

Acupuncture meridians have 361 acupoints ${ }^{[28]}$. Ruling out the acupoints at the face, head, torso, and fingertip which are inconvenient to operate, 5 acupoints in each acupuncture meridian as EPD detection acupoints were selected, as follows: 1) Lung Meridian, ZhongFu (LU1), Tianfu (LU3), Chize (LU5), Kongzui (LU6), Taiyuan (LU9); 2) Large Intestine Meridian, Quchi (LI11), Wenliu (LI7), Pianli (LI6), Yangxi (LI5), Hegu (LI4); 3) Stomach Meridian, Zusanli (ST36), Shangjuxu (ST37), Xiajuxu (ST39), Chongyang (ST42), Neiting (ST44); 4) Spleen Meridian, Xuehai (SP10), Yinlingquan (SP9), Diji (SP8), Lougu (SP7), Taibai (SP3); 5) Heart Meridian, Qingling (HT2), Shaohai (HT3), Lingdao (HT4), Shenmen (HT7), Shaofu (HT8); 6) Small Intestinal Meridian, Xiaohai (SI8), Zhizheng (SI7), Yanglao (SI6), Wangu (SI4), Qiangu (SI2); 7) Bladder Meridian, Weizhong (BL40), Chengjin (BL56), Feiyang (BL58), Kunlun (BL60), Jinggu (BL64); 8) Kidney Meridian, Yingu (KI10), Zhubin (KI9), Fuliu (KI7), Taixi (KI3), Rangu (KI2); 9) Pericardium Meridian, Tianquan (PC2), Quze (PC3), Neiguan (PC6), Daling (PC7), Laogong (PC8); 10) Triple Energizer Meridian, Tianjin (TE10), Sidu (TE9), Waiguan (TE5), Yangchi (TE4), Zhongzhu (TE3); 11) Gallbladder Meridian, Yanglingquan (GB34), Guangming (GB37), Qiuxu (GB40), Zulinqi (GB41), Diwuhui (GB42); 12) Liver Meridian, Ququan (LR8), Zhongdu (LR6), Ligou (LR5), Taichong (LR3), Xingjian (LR2).

Table 1. Acupoint Selection of Bilateral Acpuncture Meridians

\section{Randomization}

The investigator assigned a computer-generated random number to each participant. Hypertension participants were randomly allocated to TAT-SOD treatment group and placebo control group with 1:1 ratio. The investigator was with no clinical involvement in the trial.

\section{Treatment protocol}

Patients have applied $0.2 \mathrm{~mL}$ of $3000 \mathrm{U} / \mathrm{ml}$ TAT-SOD cream, in an area of $1 \mathrm{~cm}^{2}$ at acupoint Zusanli (ST36) for intervention and then massaged assisted by massage stick for a minute ${ }^{[19]}$, while placebo group used the vehicle cream instead, 2 hours before meal once a day for 15 days treatments. 2 hours after lunch, the subjects were conscious, placed in a supine position, and asked to breathe calmly. The acupoints were localized according to name and location of acupoints: WHO Standard Acupoint locations in the Western Pacific Region $^{[28]}$. The hair on the selected detection acupoints was trimmed. After previously disinfected with medical alcohol, the acupoints were connected to a digital potentiometer (Chengdu yiqi, Chengdu, China) via $\mathrm{Ag} / \mathrm{AgCl}$ disposable ECG electrodes (Shanghai junkang Company, Shanghai, China). Data of the EPD 
between two acupoints in one acupuncture meridian was collected and recorded. The proximal acupoint used as positive pole, and the distal acupoint as negative pole. For each acupuncture meridian, there are 5 acupoints, made up of 10 positive-negative electrode couples. For each subject, there are 240 positive-negative electrode couples in 24 acupuncture meridians (both left and right sides). Manual blood pressure was measured using OMRON HEM-1000 intelligence sphygmomanometer (OMRON, China) 2 hours after lunch once a day.

\section{Blinding}

Both the patients and researchers conducting the study were blinded. The study intervention products were prepared centrally by the hospital pharmacy at the Hospital of Fujian Traditional Chinese Medicine University by independent pharmacist not involved in the trial. The active product and placebo were packaged in identical glass jars and labeled with participant code numbers. The appearance and texture of the products were identical. This procedure was performed by an independent pharmacist, who was the only person aware of the codes' meaning. The participant code number's meaning was concealed until all data were analyzed.

\section{Statistical analysis}

The study design called for at least 16 evaluable patients and 16 healthy subjects. Sample size estimations were not performed. Because of the complex design of this pilot study, sample size was chosen on the basis of practical considerations. Therefore this study was not designed to have sufficient power, and the results of statistical testing have to be interpreted as descriptive, explorative, and hypothesis generating rather than as confirmatory. Data are reported as means (SD). The statistical analysis was performed with SPSS 15.0 (SPSS, Inc, Chicago, Ill) and R version 2.9.0 software. Results in patients and healthy subjects were compared using two-sample $t$-test.

\section{RESULTS}

Sixteen patients ( 8 patients in TAT-SOD treatment group and 8 patients in placebo control group) and 16 healthy subjects successfully completed the whole trial. The study flow is presented in Figure 1. Patients and healthy subjects were comparable at entry for age, sex, body height, body weight, BMI (Table 1). No adverse events occurred.

Results of acupoint EPD measurements of the lowest standard deviation of healthy subjects in each acupuncture meridian are shown in Table 2. Acupoints of the lowest standard deviation in each acupuncture meridian are Source acupoint (Yuan Acupoint) and Sea acupoint (He Acupoint) couples except Stomach Meridian, Triple Energizer Meridian and Liver Meridian. Significant EPD differences between patients and healthy subjects were observed in Stomach Meridian, Spleen Meridian, Small Intestine Meridian, Kidney 
Meridian, Gallbladder Meridian and Liver Meridian. The largest EPD difference between patients and healthy subjects was the left acupoints Yanglingquan (GB34) - Qiuxu (GB40), the difference value was $39.3 \mathrm{mV}$.

Figure 2 to Figure 4 show effects of daily TAT-SOD application at acupoint Zusanli (ST36) of patients for 15 days on systolic blood pressure (SBP), diastolic blood pressure (DBP) and EPD of the left acupoints Yanglingquan (GB34) - Qiuxu (GB40). Daily application of TAT-SOD at ST36 for 15 days on essential hypertension patients significantly decreased SBP and DBP of $179.6 \mathrm{mmHg}$ and $81.5 \mathrm{mmHg}$ to $153.1 \mathrm{mmHg}$ and $74.1 \mathrm{mmHg}$, respectively. Responding to the change in blood pressure, EPD between the left acupoints of Yanglingquan (GB34) and Qiuxu (GB40) also declined from $44.4 \mathrm{mV}$ to $22.8 \mathrm{mV}$ with the same trend. Daily application of placebo cream the patients resulted in no change in SBP, DBP and EPD of the left acupoints Yanglingquan (GB34) - Qiuxu (GB40).

\section{DISCUSSION}

For healthy subjects, EPD of Source-Sea (Yuan-He) acupoint pairs in most meridians showed the lowest standard deviation with the optimum reproducibility. In traditional Chinese medicine, Source (Yuan) means origin, and refers to the source of bio-energy (Qi), while Sea (He) acupoints are where the bio-energy (Qi) of the meridian collects and goes deep into the body. Source acupoints can reflect the visceral disease and Sea acupoints are known to be the target for effective treatment of rebellious bio-energy (Qi). Wang ${ }^{[29]}$ discovered the combined acupuncture of source and sea acupoints activated and decreased the multiple brain regions of "splanchnic brain". The outstandingly high reproducibility of EPDs between Source-Sea (Yuan-He) acupoint pairs shown in table 2 implies the outstandingly high interfacial conductivity of Yuan-He acupoints. Those results not only suggest the pair can be selected as EPD detection sites with a high reliability, but also proves the TCM observations about the uniqueness of Source-Sea (Yuan-He) acupoints.

The EPD of patients and healthy subjects were significantly different in Stomach Meridian, Spleen Meridian, Small Intestine Meridian, Kidney Meridian, Gallbladder Meridian and Liver Meridian. The first three EPDs with the largest differences between patients and healthy subjects were the left acupoints Yanglingquan (GB34) - Qiuxu (GB40) in Gallbladder Meridian, the left acuponts Xiajuxu (ST39) - Neiting (ST44) in Stomach Meridian and the right acuponts Yinlingquan (SP9) - Taibai (SP3) in Spleen Meridian, the difference values were $39.3 \mathrm{mV}, 23.8 \mathrm{mV}$ and $17.8 \mathrm{mV}$. Accordingly, the left acupoints Yanglingquan (GB34) - Qiuxu (GB40) was screened out for the EPD detection. Gallbladder Meridian, Stomach Meridian and Spleen Meridian are considered to be most effective in treating essential hypertension ${ }^{[21]}$. Acupoint Zusanli (ST36) was therefore selected for interventions with topical application of $0.2 \mathrm{ml}$ of TAT-SOD cream once a day for 15 days. 
The enzymatic scavenging of the intracellular superoxide at acupoint Zusanli (ST36) significantly decreased SBP and DBP. As shown in Figure 2 and 3, at the beginning, patients' SBP and DBP was $179.6 \mathrm{mmHg}$ and $81.5 \mathrm{mmHg}$; at the 15th day, they were declined to $153.1 \mathrm{mmHg}$ and $74.1 \mathrm{mmHg}$, respectively. The placebo group has no significant change. Acupuncture and massage at acupoint Zusanli (ST36) are reported to be effective in the treatment of essential hypertension ${ }^{[21,30]}$. The enzymatic scavenging of the intracellular superoxide at acupoints in the clinical setting achieved an efficacy in treating essential hypertension patients comparable to acupuncture. It further proves that the enzymatic scavenging of intracellular superoxide could generate acupuncture-like efficacy as was shown in the treatments of simple obesity ${ }^{[10,11]}$ and allergic rhinitis $^{[12]}$.

Acupuncture has been proved to be effective, but other than scientifically mysterious bio-energy or Qi concept, there is hardly any clue to what exactly happens immediately after a needle is inserted into acupoints in modern scientific terms, not to mention the whole mechanism of the acupuncture therapy. On the contrary, TAT-SOD can do nothing but to scavenge intracellular superoxide anions once it is applied topically to acupoints. The results in Figure 2 and 3 suggested that intracellular removal at acupoints may be the same mechanism for acupuncture, reconfirming the critical involvement of superoxide anions in the meridians system.

Some studies showed that there may be changes in bio-energy and in the electrical properties of meridians response to acupuncture at acupoints ${ }^{[31,32]}$. A large amount of evidence showed that superoxide played a central role in essential hypertension pathophysiology ${ }^{[23,24]}$. The remarkably good correlation between blood pressure readings and the acupoints Yanglingquan (GB34) - Qiuxu (GB40) EPD readings was demonstrated in healthy subjects and essential hypertensive patients as well as in the course of the antioxidant intervention in this study. It implies that EPD may be an excellent indicator of superoxide status inside meridians system due to the electric nature of superoxide. The antioxidant interventions with the TAT-SOD cream at acupoint Zusanli (ST36) was reported to result in remarkable decrease in EPD between Xiajuxu (ST39) and Neiting (ST44) ${ }^{[19]}$, in which the intervention and EPD changes occurred in the same meridian. It may be suggested that the intervention in Stomach meridian could be observed precisely in the EPD measurement in Gall bladder meridian (Figure 4).

It is still difficult to determine where superoxide originates, how it is transported along the meridian line to influence the electrical properties of meridians, and why it is distributed along the acupuncture meridian line, however, the association of meridian system with superoxide as is reconfirmed by this study provides a promising new clues to not only the elucidation of the mechanism of efficacy but also the discovery of an anatomically adelomorphic system in mammals. 
Similar to the acupuncture meridian theory, the authenticity and reproducibility of electrical devices to detect and monitor acupoints are being debated and viewed with considerable skepticism in the conventional biomedical community ${ }^{[4]}$. As shown in Figure 5, a mechanism is proposed to explain how superoxide removal results in a change in EPD between two acupoints along a meridian. If superoxide or superoxide like electron is travelling along meridians under a driven force of the voltage difference, the intracellular superoxide scavenging at acupoints converts superoxide anions into hydrogen peroxide and oxygen, reducing the total electric charge traveling along the meridian, which naturally leads to the decrease in EPD. With acupoints EPD determination, it is possible to monitor any forms of interventions at acupoints including acupuncture, massage and so on. After more efforts of confirmation, this simple EPD can possibly become a powerful tool to upgrade the scientific quality of acupuncture treatment and accelerate its basic research.

\section{Conclusions}

Enzymatic scavenging of the intracellular superoxide at Zusanli (ST36) proved to be effective in decreasing SBP and DBP. The reconfirmation of the invovlement of superoxide anions in meridians demonstrates that EPD between acupoints may be an indicator to reflect their transducting status. Moreover, preliminary results of a correlation between EPD readings and blood pressure readings may imply a possibility of using EPD as a sensitive parameter to determine blood pressure and to monitor the treatment.

\section{Conflict of Interests}

The authors declare that there is no conflict of interests regarding the publication of this paper.

\section{Author Contributions}

Guo J, Xu J, Chen T, Wang Q and Rao P designed the study, analysed the data, and prepared this manuscript; Guo J, Xu J, Xu M and Zhang C performed the research; Liu S, Ke L and Zhou J finished TAT-SOD expression; Guo J, Xu M and Zhang $\mathrm{C}$ researched electric properties of acpoints. All authors contributed to the scientific discussion.

\section{Acknowledgment}

We Thank Xu Jin-sen of Fujian University of Traditional Chinese Medicine, who gave us great help in the clinical trial application. This research was supported by National Key R\&D Program of China (No. 
2016YFD0400202), National Natural Science Foundation of China (No. 31500685, 30973720), Zhejiang Provincial Natural Science Foundation of China (No. LY16C050002), Start-Up Fund of Research in Quanzhou Normal University (No. G16062).

\section{REFERENCES}

1. Rong P, Zhu B, Li Y, Gao X, Ben H, Li Y, et al. Mechanism of acupuncture regulating visceral sensation and mobility. Front Med 2011; 5:151-156.

2. Voll R. Twenty years of electro-acupuncture diagnosis in Germany. A progress report. Amer J Acupun 1975; 3:7-17.

3. Nakatani Y. An aspect of the study of Ryodoraku. Clinic of Chinese Medicine 1956; 3:54.

4. Ahn AC, Martinsen OG. Electrical characterization of acupuncture points: technical issues and challenges. J Altern Complement Med 2007; 13:817-824.

5. Ahn AC, Park M, Shaw JR, McManus CA, Kaptchuk TJ, Langevin HM. Electrical impedance of acupuncture meridians: the relevance of subcutaneous collagenous bands. PLoS One. 2010; 5:e11907.

6. Lorenzen I, Mullen L, Bekeschus S, Hanschmann EM. Redox Regulation of Inflammatory Processes Is Enzymatically Controlled. Oxid Med Cell Longev 2017; 2017:8459402.

7. Dolowschiak T, Chassin C, Ben Mkaddem S, Fuchs TM, Weiss S, Vandewalle A, et al. Potentiation of epithelial innate host responses by intercellular communication. PLoS Pathog. 2010; 6:e1001194.

8. Bechtel W, Bauer G. Modulation of intercellular ROS signaling of human tumor cells. Anticancer Res 2009; 29:4559-4570.

9. Guo J, Liu S, Cheng X, Zhou J, Ke L, Chen X, et al. Revealing acupuncture meridian-like system by reactive oxygen species visualization. Biosci Hypotheses 2009; 2:443-445.

10. Guo J, Chen Y, Yuan B, Liu S, Rao P. Effects of intracellular superoxide removal at acupoints with TATSOD on obesity. Free Radic Biol Med 2011; 51:2185-2189.

11. Moore K, Roberts LJ. Effects of intracellular superoxide removal at acupoints with TAT-SOD on obesity. Free Radic Biol Med 2011; 51:2163.

12. Guo J, Xu M, Zheng M, Liu S, Zhou J, Ke L, et al. Topical Application of TAT-Superoxide Dismutase in Acupoints LI 20 on Allergic Rhinitis. Evid Based Complement Alternat Med 2016; 2016:3830273.

13. Ye N, Liu S, Lin Y, Rao P. Protective effects of intraperitoneal injection of TAT-SOD against focal cerebral ischemia/reperfusion injury in rats. Life Sci 2011; 89:868-874.

14. Zhang C, Chen X, Liu S, Liu Y, Rao P. Protective and repairing abilities of fusion protein PTD-SOD for L-02 cell irradiated by UV-C. J Radiat Res Radiat Process 2007; 25:248-251. 
15. Pan J, Peng S, Zhou J, He H, Liu S, Rao P. Protective Effects of Recombinant Protein PTD-SOD on Guinea Pigs Damaged by Ultraviolet B. Radiat Prot 2010; 30:284-288.

16. Zhao H, Pan J, Liu S, Zhang C, Rao P. Membrane transduction activity of TAT-SOD fusion protein. J Fuzhou Univ 2007; 35:936-940.

17. Zhao H, Zhang C, Liu Y, Liu S, RaoP. PTD-SOD protects cells against cell injury caused by UV-B. J Fuzhou Univ 2009; 37:446-451.

18. Pan J, Zhou J, Peng S, He H, Liu S, Rao, P. The preventing effects of recombinant protein PTD-SOD on guinea pigs damaged by Ultraviolet B. J Radiat Res Radiat Process 2009; 27:297-302.

19. Xu M, Guo J, Xu J, Zhang C, Liu S, Liao R, et al. Monitoring the Effects of Acupoint Antioxidant Intervention by Measuring Electrical Potential Difference along the Meridian. Evid Based Complement Alternat Med 2015; 2015:286989.

20. Xu M, Guo J, Ni L, Rao P, Chen H, Chen Y. The Effect of Infusions of Tea Soup on Electrical Potential Difference Along the Meridian. Journal of Chinese Institute of Food Science and Technology 2018; 18:27-33.

21. Tam KC, Yiu HH. The effect of acupuncture on essential hypertension. Am J Chin Med (Gard City N Y) 1975;3:369-375.

22. Zhang Y, Du Y. Acupuncture combined with medication for morning blood pressure of essential hypertension. Zhongguo Zhen Jiu 2018; 38:343-348.

23. González J, Valls N, Brito R, Rodrigo R. Essential hypertension and oxidative stress: New insights. World J Cardiol 2014; 6:353-366.

24. Wang Y, Li Y, Zhou L, Guo L. Protective effects of electroacupuncture at LR3 on cardiac hypertrophy and apoptosis in hypertensive rats. Acupunct Med 2016; 34:201-208.

25. Severcan C, Cevik C, Acar HV, Sivri AB, Mit SS, Geçioğlu E, et al. The effects of acupuncture on the levels of blood pressure and nitric oxide in hypertensive patients. Acupunct Electrother Res 2012; 37:263275.

26. Chen H, Huang X, Chen C, Liu S, Rao P. Effect of Shaking-flask Conditions on Expression of PTD-SOD by Escherichia Coli. J Med Theor Prac 2006; 19: 1255-1257.

27. Sung J, Han KH, Zo JH, Park HJ, Kim CH, Oh BH. Effects of red ginseng upon vascular endothelial function in patients with essential hypertension. Am J Chin Med 2000; 28:205-216.

28. World Health Organization Regional Office for the Western Pacific. WHO Standard acupuncture point locations in the Western Pacific Region. Geneva: World Health Organization, 2008. 
29. Wang GB, Liu C, Wu LB, Yan B, Gao SZ, Shao GR, et al. Functional magnetic resonance imaging on acupuncturing Yuan-Source and He-Sea acupoints of stomach Meridian of Foot-Yangming. Zhongguo Yi Xue Ke Xue Yuan Xue Bao 2009; 31:171-176.

30. Yang X, Zhao H, Wang J. Chinese massage (Tuina) for the treatment of essential hypertension: a systematic review and meta-analysis. Complement Ther Med 2014; 22:541-548.

31. Lee Y, Kim S, Son T, Kang D, Jung B. Meridian electrical potential response to acupuncture stimulation between operator and subject. J Acupunct Meridian Stud 2010; 3:249-254.

32. Khorsand A, Zhu J, Bahrami-Taghanaki H, Baghani S, Ma L, Rezaei S. Investigation of the electrical impedance of acupuncture points and non-acupuncture points before and after acupuncture, using a fourelectrode device. Acupunct Med 2015; 33:230-236.

2 Figure legends

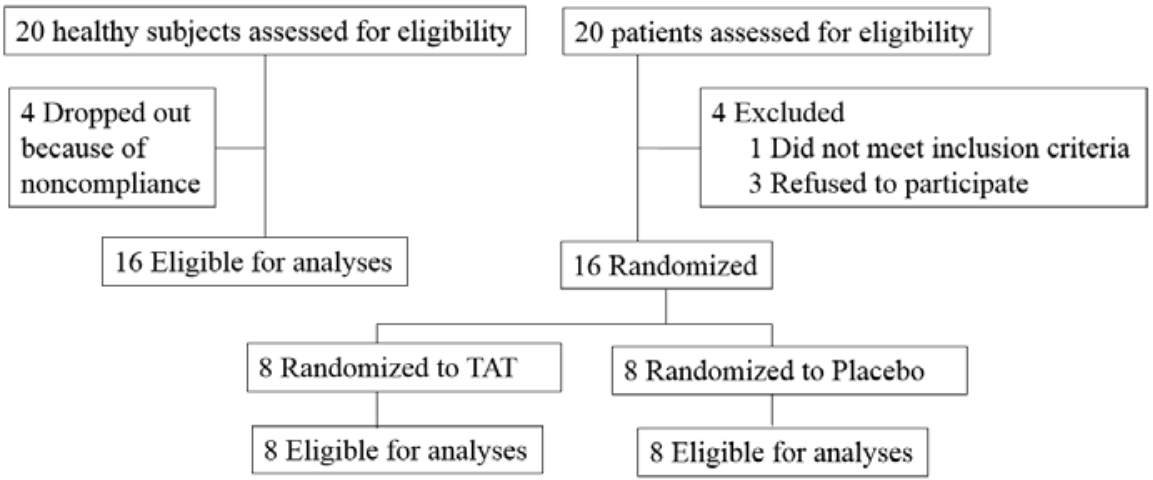

4 Figure 1. Study Flow Chart.

5 


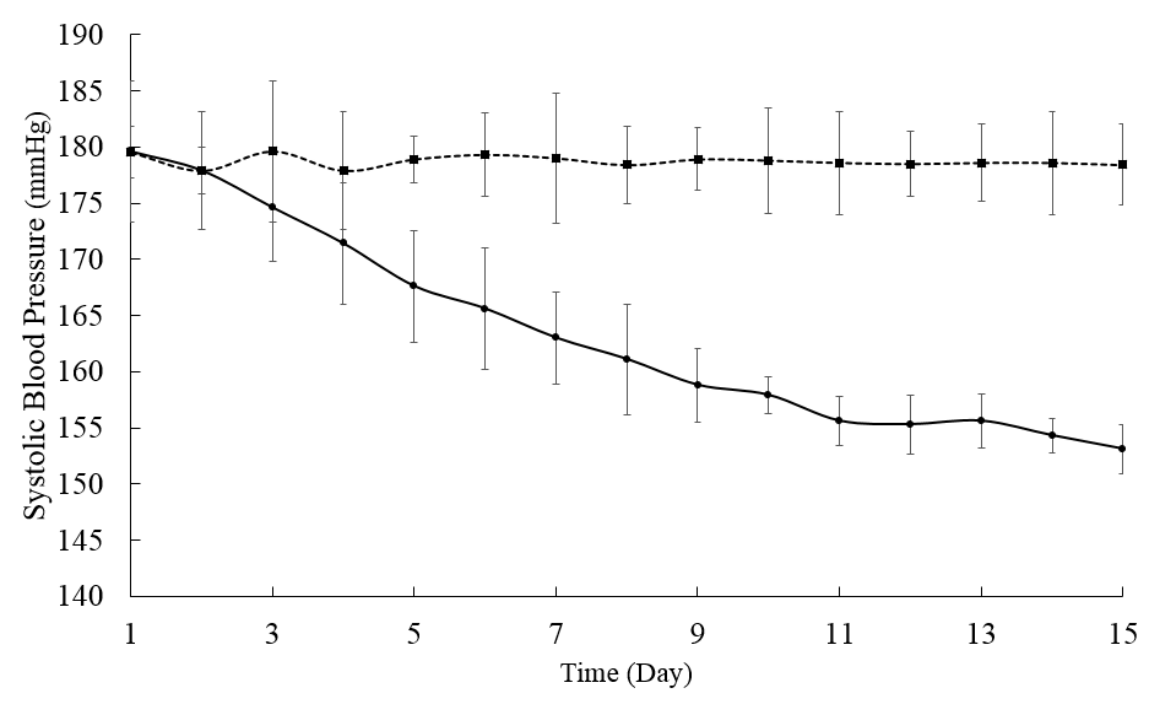

$$
\rightarrow \text { TAT-SOD } \rightarrow--\cdot P l a c e b o
$$

Figure 2. Monitoring the SBP of Essential Hypertension Patients Caused by Acupoint Zusanli (ST36) 8 TAT-SOD Interventions.

9

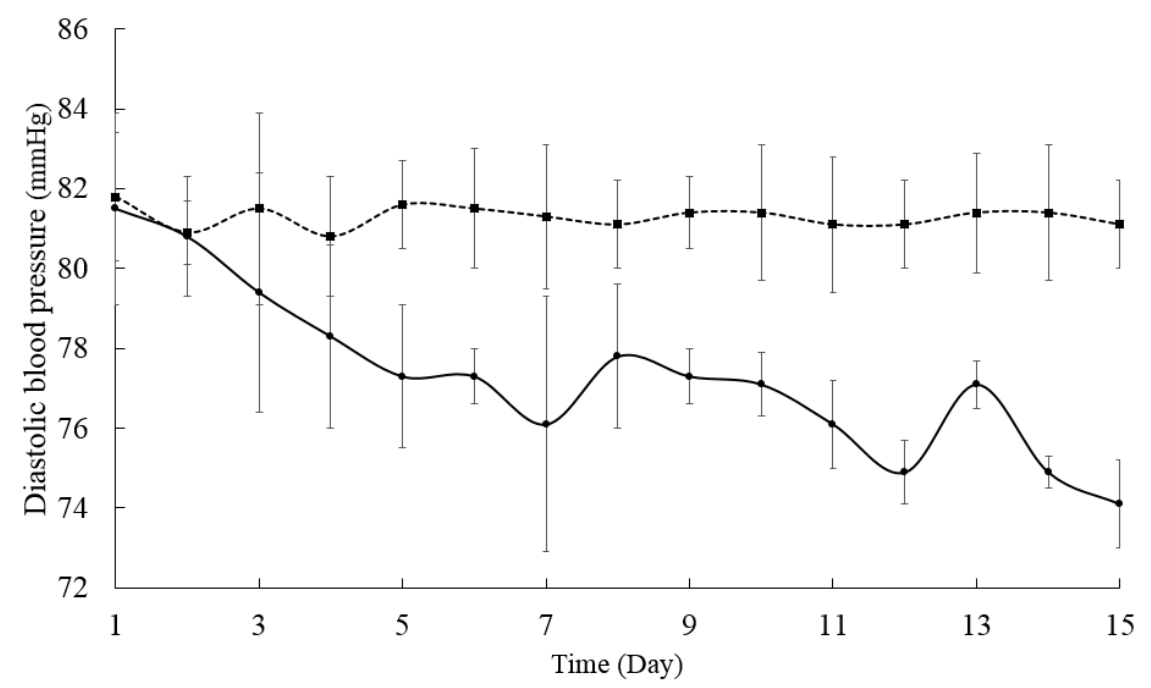

$$
\rightarrow \text { TAT-SOD } \rightarrow-\text { Placebo }
$$

Figure 3. Monitoring the DBP of Essential Hypertension Patients Caused by Acupoint Zusanli (ST36) TAT-SOD Interventions. 


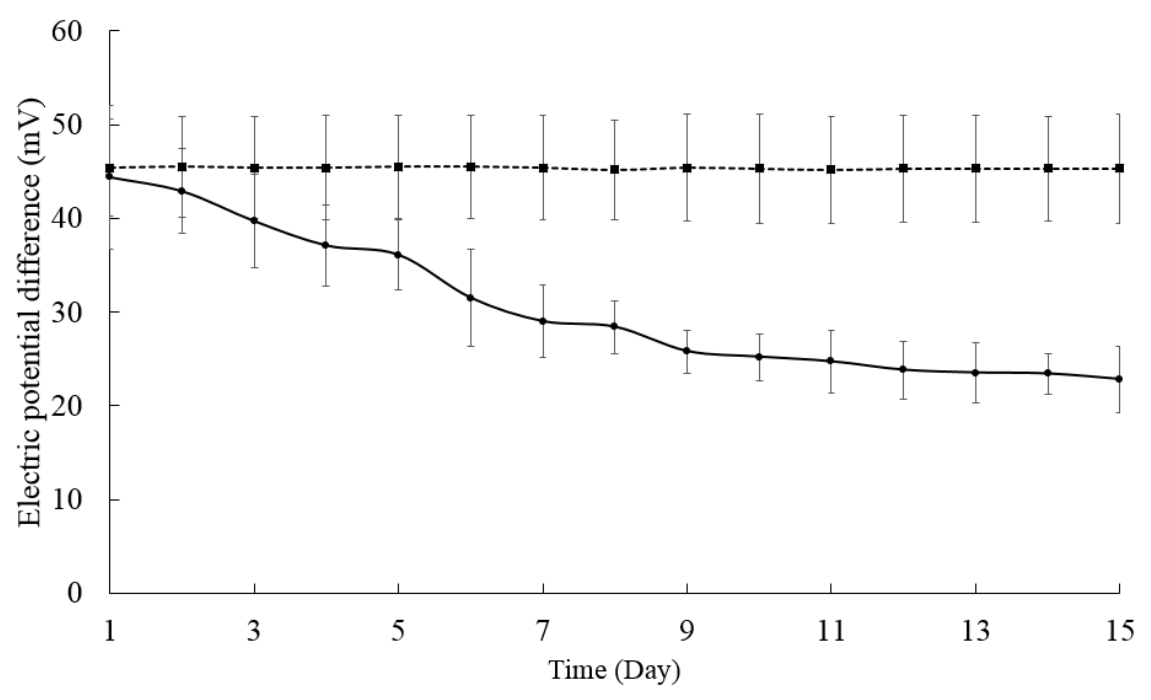

$$
\rightarrow \text { TAT-SOD } \rightarrow-\text { Placebo }
$$

Figure 4. Monitoring the acupoints Yanglingquan (GB34) - Qiuxu (GB40) Electric Potential Difference

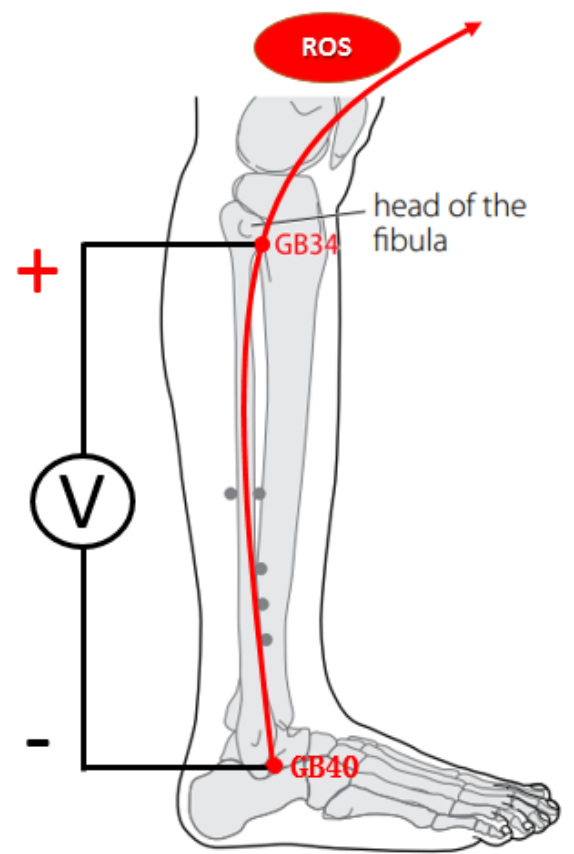

Figure 5. A proposed mechanism about the superoxide removal at acupoint Zusanli (ST36) and the resultant EPD change between GB30 and 40.

23 Table 1. Clinical Information on All Subjects 


\begin{tabular}{llll}
\hline Parameter/treatment & Patients & Healthy subjects & p-values \\
\hline $\mathrm{N}$ & 16 & 16 & \\
Age (year) & $54.3 \pm 7.8$ & $52.1 \pm 6.9$ & 0.53 \\
Sex (M/F) & $9 / 7$ & $8 / 8$ & $\mathrm{NA}$ \\
Body height (m) & $1.66 \pm 0.09$ & $1.66 \pm 0.07$ & 0.97 \\
Body weight (kg) & $69.8 \pm 12.9$ & $66.1 \pm 14.0$ & 0.67 \\
BMI & $25.2 \pm 2.3$ & $23.8 \pm 3.2$ & 0.33 \\
Course of disease (month) & $61.0 \pm 10.9$ & $\mathrm{n} / \mathrm{a}$ & $\mathrm{NA}$ \\
PR (bpm) & $75.0 \pm 4.0$ & $62.5 \pm 5.0$ & $<0.01$ \\
SBP (mmHg) & $179.6 \pm 4.6$ & $111.3 \pm 4.6$ & $<0.01$ \\
DBP (mmHg) & $81.6 \pm 2.0$ & $62.5 \pm 5.0$ & $<0.01$ \\
\hline
\end{tabular}

24 F, Female; M, male.

25 NA, not applicable.

26

Table 2. The Electric Potential Difference of Acupoints along 12 Acupuncture Meridians

\begin{tabular}{cccccc}
\hline \multirow{2}{*}{ Meridians } & \multirow{2}{*}{ Acupoints } & \multicolumn{2}{c}{ Electric potential difference (mV) } & \multirow{2}{*}{ Difference (mV) } & \multirow{2}{*}{ p-values } \\
& & Patients & Healthy subjects & & \\
\hline Lung & LU5-LU9 (Right)* & $-15.4 \pm 4.8$ & $-20.8 \pm 2.8$ & 5.4 & 0.02 \\
Large Intestine & LI11-LI4 (Left)* & $0.7 \pm 2.4$ & $1.7 \pm 2.4$ & -1.0 & 0.36 \\
Stomach & ST39-ST44 (Left) & $38.6 \pm 3.4$ & $14.8 \pm 1.1$ & 23.8 & $<0.01$ \\
Spleen & SP9-SP3 (Right)* & $5.1 \pm 7.2$ & $-12.7 \pm 4.3$ & 17.8 & $<0.01$ \\
Heart & HT3-HT7 (Right)* & $0.4 \pm 4.1$ & $3.1 \pm 1.5$ & -2.7 & 0.11 \\
Small Intestine & SI8-SI4 (Left)* & $-0.4 \pm 1.2$ & $-6.4 \pm 1.2$ & 6.0 & $<0.01$ \\
Bladder & BL40-BL64 (Right)* & $9.5 \pm 8.6$ & $8.5 \pm 3.6$ & 1.0 & 0.76 \\
Kidney & KI10-KI3 (Left)* & $-9.1 \pm 4.9$ & $3.7 \pm 3.5$ & -12.8 & $<0.01$ \\
Pericardium & PC3-PC7 (Left)* & $-2.4 \pm 4.6$ & $-2.6 \pm 1.4$ & 0.2 & 0.91 \\
Triple Energizer & TE9-TE5 (Left) & $-0.1 \pm 5.5$ & $-2.0 \pm 3.1$ & 1.9 & 0.39 \\
Gallbladder & GB34-GB40 (Left)* & $44.9 \pm 6.4$ & $5.6 \pm 0.9$ & 39.3 & $<0.01$ \\
Liver & LR6-LR5 (Left) & $-5.7 \pm 8.2$ & $5.9 \pm 3.2$ & -11.6 & $<0.01$ \\
\hline
\end{tabular}

$28 *$ Source-Sea (Yuan-He) acupoint couples. 